\title{
Perception et autoportrait de l'interprète indigène en Afrique coloniale française
}

\author{
Raymond Mopoho \\ Dalhousie University
}

\begin{abstract}
African interpreters were hired to serve as intermediaries between Europeans and Africans, but they ended up establishing themselves as key parties in the mediation process, wielding as much power as both the colonial administrators and the traditional authorities. In so doing, they actively participated in the colonial enterprise, which involved dominating and exploiting native masses, promoting Eurocentrism, as well as fostering the rule of injustice and violence. Although in the African community the interpreter's status brought him privileges and some respect, he was viewed with suspicion - and even contempt - by European colonial officers, who considered him as a threat to their own existence. Eventually, this indirect actor of the disintegration of African traditional societies could really identify neither with his fellow natives, for whom he was part of the colonial administration, nor with Europeans, who would rather keep him in a state of servitude. His personality reflected the contradictions of the new social order which he had helped to establish.
\end{abstract}

\section{Introduction}

L'apparition du métier d'interprète en Afrique est une conséquence directe de l'implantation des régimes coloniaux sur le continent. Cette implantation est précédée de nombreuses missions d'exploration visant "à étudier les pratiques commerciales des indigènes, les possibilités d'exploitation commerciale du territoire, la collecte de données géologiques, topographiques, géographiques, ethnographiques, ainsi que sur les ressources minières et diverses autres richesses des territoires explorés" (Raffenel 1846: s.p.). La colonisation s'accompagne de nombreux bouleversements sur le plan sociopolitique. Par exemple, les autorités traditionnelles sont dépouillées de leur pouvoir et deviennent de simples exécutants des ordres du commandant (administrateur colonial et, par extension, tout Européen ou assimilé en colonie). De même, l'ascension sociale ne dépend plus de l'héritage ou d'autres critères traditionnels, mais du degré de maîtrise de la langue française et des fonctions que l'on remplit au sein de l'administration coloniale. C'est qu'en raison d'une pénurie chronique de personnel européen, tous les autochtones lettrés qui le désirent sont recrutés dans la fonction publique, comme cadres indigènes. Parmi ces derniers, l'interprète occupe une place de choix: attaché au service du commandant local, il est l'Africain le plus proche du centre du pouvoir (voir Mopoho 2001 pour davantage de détails). 
Officiellement recruté pour traduire oralement les échanges entre le colon et l'autochtone, l'interprète assume invariablement des fonctions plus importantes de médiateur et de conseiller auprès des interlocuteurs, intervenant tantôt pour le compte de l'une des parties, tantôt pour celui de l'autre. Sa sphère d'influence s'étend à tous les secteurs et à toutes les couches de la société coloniale. L'interprète apparaît comme la véritable cheville ouvrière de l'administration coloniale. Cependant, pour crucial qu'il soit, son rôle est rarement reconnu. Bien au contraire, la légende populaire, relayée entre autres par les œuvres littéraires et les rapports des administrateurs coloniaux, donne de l'interprète un portrait peu flatteur, celui-ci apparaissant généralement comme un individu corrompu, manipulateur et incompétent. On peut par exemple lire dans Manga Mado (1970: 50):

De deux ou trois phrases de son chef, il faisait tout un sermon plein de menaces, d'injures, et exagérait l'impossible, commandait l'inexécutable: bref, l'interprète "interprétait" [et] ne traduisait pas. On n'a jamais su si le chef "Blanc" savait vraiment tout ce que tramait ce petit homme grincheux, trahi qu'il était par son manque d'expérience professionnelle.

Dans le présent article, nous nous essaierons plutôt à décrire l'auto-image de l'interprète, c'est-à-dire tel qu'il se voit lui-même, et ce à travers une analyse de ses rapports avec les diverses composantes de la société coloniale. À cette fin, nous nous servirons de deux récits autobiographiques, à savoir L'étrange destin de Wangrin (Hampaté 1992) et Doigts noirs (Moukouri 1963).

\section{Présentation sommaire des deux ouvrages}

\subsection{L'étrange destin de Wangrin}

Lorsque cet ouvrage paraît en 1992, il est unanimement salué comme un chef-d'œuvre de reproduction de la littérature orale africaine, dans lequel l'auteur donne toute la mesure de ses immenses talents de conteur. Quelques critiques s'interrogent toutefois sur l'authenticité aussi bien du héros que du récit. Dans la postface de la deuxième édition de l'ouvrage, l'auteur répond comme suit à ces interrogations:

Depuis la parution de ce livre en 1973, certains malentendus sont apparus çà et là tant sur la personnalité réelle du héros que sur la nature même de l'ouvrage. Je ne sais pas pourquoi certains ... s'interrogent: ce récit est-il une fiction, une réalité, ou un habile mélange des deux? On admet généralement l'existence historique de celui qui s'était surnommé lui-même "Wangrin", mais on pense que j'ai dû "romancer" quelque peu sa vie, y introduisant même, pour corser l'histoire et lui donner une sorte de signification symbolique, un dosage subtil de tradition orale et d'événements surnaturels de mon cru ... Mais si tel était bien le cas, pourquoi le nierais-je? ... Et pourquoi aurais-je pris la précaution de bien préciser en tête de l'ouvrage que tous les personnages et les événements du livre étaient authentiques? (Hampaté 1992: 359) 
Nous considérerons quant à nous que cet ouvrage est une fidèle traduction/transcription du récit de la vie professionnelle de Wangrin, tel que raconté par ce dernier à l'auteur. Wangrin est un ancien élève de l'école des otages où les chefs et les notables envoient "de gré ou de force" (Hampaté 1992: 18) leurs fils, en gage de fidélité aux autorités coloniales. Après l'obtention de son certificat d'études primaires indigènes, soit le plus haut diplôme qu'un autochtone soit autorisé à acquérir, il entre dans les cadres subalternes de l'administration coloniale, en qualité de moniteur de l'enseignement primaire. Au bout d'un an de service, bien que s'étant acquitté de manière remarquable de ses fonctions d'enseignant, Wangrin est profondément insatisfait de sa situation socio-économique. Par rapport aux autres membres de l'élite autochtone occidentalisée, il n'est guère qu'un fonctionnaire marginal, sans influence ni renommée, et sa solde ne lui permet pas de mener le genre de vie dont il rêve. Sa frustration est d'autant plus grande que l'interprète Racoutié, dont les connaissances en français sont des plus rudimentaires, est le deuxième personnage de la ville après le commandant. Quoique quasi illettré, Racoutié exerce, de par ses fonctions, une grande influence jusque sur le commandant lui-même. Il vit dans l'opulence et il est sollicité pour toutes les affaires importantes de la ville. Il a une très grande idée de l'importance que lui confèrent ses fonctions, et il n'hésite pas à prendre la relève des griots pour chanter ses propres louanges en narguant ses adversaires:

Je suis Racoutié, ancien sergent de Fantirimori ... Je suis présentement l'interprète du commandant. Je suis son œil, son oreille et sa bouche. Chaque jour, je suis le premier et le dernier auxiliaire qui le voit. Je pénètre dans son bureau à volonté. Je lui parle sans intermédiaire ... Je suis Racoutié qui s'assied sur un banc en beau bois de caïlcédrat devant la porte du commandant blanc. Qui parmi vous ignore que le commandant a droit de vie et de mort sur nous tous? Que ceux qui l'ignorent sachent que ma bouche, aujourd'hui, Dieu merci, se trouve être la plus proche de l'oreille du commandant. (45)

Racoutié n'éprouve que du mépris pour Wangrin qui, bien qu'instruit, exerce une profession dont il ne tire de toute évidence ni privilège ni honneur: "Que penser d'un adulte qui passe son temps à courir, sauter, danser, chanter avec des gamins, sinon qu'il est lui-même ou bambin ou crétin?" (38), s'étonnet-il. Convaincu de sa toute-puissance, il agresse physiquement Wangrin, ce qui entraîne sa chute: l'enquête diligentée par les autorités établit sa responsabilité dans l'agression, et pour ne pas perdre la face, il prend l'initiative de se faire muter ailleurs. Wangrin est immédiatement recruté pour le remplacer à titre d'abord provisoire, puis définitif quand son transfert dans le cadre des interprètes est entériné par la haute administration. Lors de sa prise de service en tant qu'interprète, son patron définit comme suit la tâche - et le comportement - qu'il attend de lui:

Il va falloir que tu ouvres œil et oreille afin de bien voir, enregistrer et renseigner exactement ton commandant de cercle. C'est un devoir 
péremptoire pour un homme instruit comme toi. Mon vieil idiot d'interprète me fut imposé. Il a été l'ordonnance de je ne sais plus quel officier de la conquête. Il est très fat avec ses frères de race et obséquieux avec les Blancs. Je n'aime pas beaucoup cette attitude. (33)

N'étant pas originaire de la région, Wangrin sait qu'il ne peut s'acquitter convenablement de sa mission que s'il jouit, par ailleurs, de la confiance des autochtones. Aussi adhère-t-il au waaldé (association de classe d'âge) de son quartier. Son appartenance à ce waaldé, le plus puissant de la ville, lui permet en outre de pénétrer les secrets de l'organisation en vue de renseigner ses supérieurs. En plus de servir d'interprète au commandant, Wangrin s'occupe de son secrétariat, ainsi que de la distribution du courrier, de la préparation des dossiers relatifs aux litiges, et du maintien des archives. Il rend également de menus services personnels au commandant. Il mène désormais un train de vie digne de la haute personnalité qu'il estime être: il s'aménage une grande résidence où il offre de somptueux repas et reçoit d'innombrables visiteurs (ceux-ci lui apportent des cadeaux, des pots-de-vin ou encore des informations). Pour se tenir au courant de tout ce qui se passe dans la région, il établit un immense réseau de renseignement dont les informateurs sont entretenus à ses propres frais. Pendant la Première Guerre mondiale, Wangrin est chargé de la tenue des registres des réquisitions et de la rédaction des formulaires de fourniture. Avec l'aide d'un comparse, il réquisitionne frauduleusement plus de bœufs qu'autorisé, le troupeau excédentaire étant écoulé à l'étranger. Ces détournements sont facilités par le fait que Villermoz (l'adjoint au commandant), flemmard, a esquivé ses responsabilités en signant à Wangrin des réquisitions en blanc. Suite à une dénonciation, une enquête est ouverte sur "l'affaire des bœufs". Mis en cause et suspendu temporairement de ses fonctions, Wangrin essaie de se protéger en incriminant Villermoz, et pour cause:

il savait ... qu'une affaire dans laquelle un Européen se trouvait justement ou injustement impliqué serait bien difficile à trancher à la colonie. Sans doute préférerait-on étouffer n'importe quel crime plutôt que de condamner un Européen, à plus forte raison si ce dernier était un agent de l'autorité. Il en allait du prestige des colonisateurs, et la politique menée en ce domaine ne s'embarrasse pas de problèmes de conscience. (81)

Effectivement, une fois la négligence de Villermoz établie, le dossier est classé et Wangrin peut réintégrer son poste. En sortant indemne de cette affaire, Wangrin accroît sa popularité auprès d'une bonne partie de la population, tout en s'attirant l'animosité des Européens, à commencer par le nouveau commandant qu'il trouve en place à son retour. Enhardi par cette première 'victoire', Wangrin multiplie méfaits et délits (braconnage, trafic d'alcool, exercice illégal de la profession de commerçant, détournement de biens d'autrui, abus de confiance, extorsion, chantage, faux en écriture...). Ses victimes se comptent aussi bien parmi les Européens que chez les chefs traditionnels et les notables des villes et régions où il travaille. C'est ainsi qu'il réussit à amasser une immense fortune en l'espace de quelques années. 
Parvenu au faîte de sa gloire, il présente des signes de stress et finit par s'adonner à l'alcoolisme, qui le conduit à la ruine. Il n'est plus qu'un clochard au moment de sa mort.

\subsection{Doigts noirs}

Si le portrait de l'interprète Wangrin est celui d'un fonctionnaire affairiste, davantage préoccupé par l'accumulation de biens matériels, et quelque peu rebelle vis-à-vis des colons, celui de Moukouri nous révèle un agent modèle qui se dévoue, à la limite de l'abnégation, au service de l'administration coloniale. Tout comme Wangrin, Moukouri commence sa carrière dès l'obtention du certificat de fin d'études primaires. Il est nommé directement aux fonctions d'interprète. À ce titre, il devient, malgré son jeune âge (19 ans), un grand personnage dans sa famille, son village et sa région, et il fait partie de l'élite autochtone du pays. Officiellement, il sert de porte-parole et d'agent de renseignement au commandant. Mais officieusement, il est l'homme à tout faire de son employeur: secrétaire, archiviste, cantonnier, surveillant de travaux, peintre en bâtiment, jardinier... Pendant les tournées, il peut arriver que le commandant fasse de lui son cuisinier, garde de corps, chasseur, et même "entremetteur". À l'instar de tous les fonctionnaires autochtones, l'interprète a un statut à la fois fragile et flou dans l'administration coloniale: s'il est astreint à de nombreuses obligations professionnelles, ses droits sont par contre rarement reconnus. Il est tout à la merci du commandant, lequel peut à volonté lui infliger des sanctions disciplinaires, voire le renvoyer, sous le moindre prétexte. Appelé le plus souvent à jouer le rôle d'intermédiaire, son efficacité repose sur une bonne connaissance de la région, notamment son histoire, ses principaux acteurs traditionnels et les rapports qu'ils entretiennent entre eux et avec l'administration coloniale, les importants événements de la vie quotidienne, les antécédents politiques et sociaux, etc. Pour se tenir informé, il doit entretenir (comme Wangrin), par ses propres moyens, un important réseau de renseignement.

Pendant la Deuxième Guerre mondiale, la mobilisation des citoyens français provoque une pénurie aiguë de personnel européen et ouvre la voie à l'africanisation des cadres. C'est dans ce contexte que Moukouri se voit confier certaines tâches jusque-là réservées aux commandants: il doit assurer le contrôle du marché et de la prison, le recouvrement des impôts, et l'audience des affaires secondaires. Cette promotion à de plus hautes responsabilités ne s'accompagne toutefois pas d'un réaménagement de sa rémunération. Il ne bénéficie pas non plus de l'appui logistique et des divers avantages (logement, personnel de soutien, etc.) normalement liés à ces fonctions lorsque celles-ci sont occupées par un Européen. Il fait néanmoins preuve de zèle afin de mériter la 'confiance' ainsi placée en lui par ses supérieurs. Il est constamment hanté par la peur d'échouer, car cela aurait une incidence négative, selon lui, sur la carrière de tous les fonctionnaires 
autochtones. Grâce à ses nouvelles fonctions, il se sent - pour la première fois - proche de l'administration dont il épouse du reste la plupart des positions. Mais lorsque ses intérêts l'exigent, il s'associe en toute discrétion aux autochtones pour mener certaines actions, comme la création de coopératives de planteurs en vue de l'amélioration des cultures et la commercialisation des récoltes. Il semble jouer un double rôle qui n'est véritablement apprécié ni de l'élite autochtone ni de l'administration, et il s'estime mal compris.

Sur le plan professionnel, sa carrière évolue de manière fulgurante. Après la guerre, avec l'accélération de l'africanisation des cadres (et la création d'un cadre commun pour l'administration coloniale, réunissant fonctionnaires Européens et Africains), Moukouri est promu au rang d'administrateur. Il sera ensuite nommé au cabinet du ministre secrétaire d'État à la France d'outre-mer, ce qui l'amène à s'établir temporairement à Paris où il remplira les fonctions d'attaché parlementaire du ministre. Il profite de son séjour en France pour suivre des cours à l'École nationale d'outre-mer où sont formés les administrateurs coloniaux. Il rentre au Cameroun au bout de trois ans et poursuit sa carrière d'administrateur dans diverses régions du pays. En 1958, il quitte l'administration coloniale française pour la toute nouvelle administration nationale camerounaise, où il occupe le poste de chef de cabinet du ministre des Affaires économiques. Il est nommé préfet de la capitale (Yaoundé) l'année suivante; c'est le poste qu'il occupe quand survient l'indépendance du pays en 1960. Il est alors nommé premier ambassadeur du Cameroun en France et dans les pays de la Communauté. Pour lui, cette nomination marque la fin de sa carrière d'interprète (Moukouri 1963: 201). Il servira aussi comme ambassadeur au Canada avant de prendre sa retraite.

\section{L'interprète et les autochtones}

Les rapports qu'entretient l'interprète avec la communauté autochtone sont fort complexes, essentiellement emprunts de méfiance, d'opportunisme, de collaboration, d'exploitation mutuelle, et même de trahison. Quelles que soient ses origines et son statut dans la hiérarchie sociale traditionnelle, l'interprète fait d'emblée partie, comme tous les autres autochtones dits évolués (c'est-à-dire exposés à la civilisation, grâce à la scolarisation), de l'élite émergente qui est appelée à jouer les premiers rôles dans la nouvelle société coloniale. Il est à la fois l'un des symboles de l'effondrement de la société traditionnelle et l'une des incarnations du nouvel ordre établi par la colonisation. De manière générale, il jouit d'une grande notoriété auprès de l'ensemble de la population, et ce pour plusieurs raisons: il est capable de lire et d'écrire en français, aussi estime-t-on qu'il a dompté le génie du blanc et qu'il peut par conséquent lutter à armes égales avec ce dernier; par ailleurs, de par ses fonctions, l'interprète est le plus proche collaborateur autochtone de l'administrateur Européen, et la population compte sur lui pour la 
défendre et pour veiller à ses intérêts. Il s'ensuit que l'interprète exerce une grande influence sur les hommes et sur les événements de sa région. Cela est vrai de l'interprète Racoutié, tel que mentionné ci-dessus, mais aussi de l'interprète Romo Sibédi, lequel avait passé toute sa vie à "servir les Européens et leur obéir comme un robot - ce qui d'ailleurs ne l'empêchait pas d'obtenir d'eux ce qu'il voulait, sans qu'ils s'en doutassent..." (Hampaté 1992: 114). En ce qui concerne Wangrin, si l'administrateur est considéré comme le dieu de la brousse, il n'est rien de moins qu'un demi-dieu:

La maison de Wangrin était remplie de griots, musiciens, quémandeurs des deux sexes, demandeurs et défenseurs en justice, courtisans, représentants de chefs de canton, etc. Tout ce monde avait, de bouche à oreille, appris le retour du grand "manitou" du cercle et chacun tenait à lui plaire pour éviter sa colère, gagner ses faveurs, ou simplement entrer dans ses bonnes grâces. En effet, pouvoir dire "Je suis bien avec le Grand Interprète du Grand Commandant" équivalait à un titre de noblesse et arrondissait bien des angles dans la vie courante de l'époque coloniale. (Hampaté 1992: 191)

Quant à Moukouri, en plus de sa grande influence auprès des autochtones, il possède plusieurs biens qui rehaussent son prestige: un fusil européen (qu'il met en bandoulière pour se promener au village le dimanche), une lampe Aïda, un phonographe de seconde main (dont l'achat lui a été imposé par un commandant en fin de séjour), et il lui arrive de se procurer une bouteille de vin (alors qu'il est interdit de vendre de l'alcool aux autochtones) (Moukouri 1963: 44-50). En échange de l'honneur que lui accordent les autochtones, l'interprète se doit de partager avec ceux-ci son salaire. Cela étant, si l'interprète n'est pas originaire de la région où il travaille, la population tend à se montrer suspicieuse à son égard. La majorité des autochtones le perçoivent alors comme un espion à la solde de l'administration et estiment que son salaire est un bien mal acquis. Ses rares amis se gardent de s'afficher publiquement en sa compagnie, sous peine d'être eux aussi ostracisés. Il fait l'objet de nombreuses dénonciations, souvent calomnieuses, qui aboutissent en général à une affectation disciplinaire (22).

Les relations entre l'interprète et les membres de l'élite sont tout aussi difficiles. Nombre de fonctionnaires autochtones convoitent la fonction d'interprète, en raison de son prestige et des avantages qu'elle présente. Tous ceux qui le peuvent abandonnent leurs corps d'origine pour devenir interprètes. Le premier métier de Wangrin est celui d'enseignant. Ses collègues Racoutié et Romo sont d'anciens tirailleurs. Lorsque Wangrin se présente pour la première fois au bureau du commandant de Dioussola pour prendre service, il constate en y arrivant que tous les fonctionnaires indigènes l'attendent dans la cour pour lui souhaiter la bienvenue. Il comprend alors “qu' à Dioussola, comme partout ailleurs, le grand interprète [est] un personnage de premier plan" (Hampaté 1992: 226). Mais l'opulence dans laquelle vit l'interprète n'est pas favorablement appréciée par tous. Lors d'un dîner offert par Wangrin aux fonctionnaires indigènes, son griot Kountena est agressé par l'un des invités, pour avoir osé vanter les mérites 
et la générosité de son maître en ces termes: "Frères convives, mangez, buvez, mangez et buvez encore et si demain l'envie vous prend de récidiver, revenez, vous mangerez et vous boirez. Il en est ici comme du paradis d'Allâh. Tout est toujours prêt et l'on consomme sans frais" (70). L'agresseur laisse entendre que Wangrin accumule sa fortune de façon malhonnête et ne mérite pas d'éloge. De même, le marabout Tierno Siddi, qui a pourtant prié pour Wangrin lors de "l'affaire des bœufs", refuse les cadeaux que lui rapporte Wangrin de Dakar, parce qu'il l'estime coupable (100).

Le règne de l'affairisme aidant, certains interprètes entretiennent entre eux des rapports conflictuels. Ils se battent les uns contre les autres pour occuper les postes offrant le plus d'avantages. Pendant que Wangrin se rend à Goudougaoua, son nouveau lieu d'affectation, il fait escale à Yagouwahi où Romo Sibédi, l'interprète local, organise une fête pendant toute une semaine en son honneur. Séduit par la ville et gagné par un sentiment de convoitise, Wangrin annonce sans ambages à son hôte qu'il fera tout pour prendre sa place (Hampaté 1992: 100-101). Il y parviendra, et ce sera le début d'une longue inimitié entre les deux collègues. Un autre domaine de friction potentielle entre l'interprète et le reste de l'élite autochtone est celui de l'action sociopolitique. Lorsque l'interprète jouit de la confiance des autres membres de l'élite, il joue un rôle de médiateur engagé, aidant les autochtones à présenter efficacement leurs revendications au commandant d'une part, et expliquant aux populations le bien-fondé de telle ou telle action de l'administration. Il s'impose comme un des leaders des autochtones évolués, coordonnant et finançant de sa poche leurs activités, arbitrant les différends entre eux, adoptant un comportement exemplaire de personne civilisée, et tempérant les ardeurs des nationalistes (Moukouri 1963: 23). Par contre, quand l'interprète entretient avec l'élite des rapports non harmonieux, sa tâche de médiation devient difficile et délicate: il doit garder une stricte neutralité entre le commandant et la population; il ne doit ni se prononcer sur l'action de l'administration, ni jouer le moindre rôle dans les initiatives des autochtones. Bref, il lui faut s'effacer totalement devant l'élite et se montrer discret, sous peine de devenir un bouc émissaire lorsque l'une des parties n'obtient pas ce qu'elle veut (Moukouri 1963: 24).

Le dernier groupe avec lequel l'interprète a des interactions régulières est celui des chefs traditionnels. L'interprète a, pour ainsi dire, supplanté ces derniers au sommet de la société autochtone. Pour cette raison, certains ne voient en lui qu'un usurpateur. Ils semblent collaborer avec lui malgré eux, parce qu'il leur est indispensable pour dialoguer avec le commandant, et parce qu'il est celui par qui sont transmis les messages de l'administration. Il faut dire qu'il arrive que l'interprète se substitue abusivement à l'administration et dicte sa propre volonté au chef traditionnel. Il provoque parfois des conflits dont il s'arrange ensuite pour tirer, à titre personnel, le meilleur parti. Ainsi, à l'occasion du décès du chef Brildji, lorsque le commandant demande à Wangrin ce qu'il convient de faire, ce dernier lui conseille de le dépêcher auprès des membres de la famille du défunt pour 
leur présenter les condoléances de l'administration. Mais une fois cette mission à lui confiée, Wangrin se transforme en faiseur de roi, extorquant une fortune à Karibou Sawali, le frère de Brildji et successeur attitré, soidisant pour intercéder en sa faveur auprès de l'administration qui aurait l'intention de confier la chefferie plutôt au fils de Brildji. Wangrin incite Karibou à user de violence pour laver l'affront qu'on lui aurait fait d'enterrer son frère en son absence. Rendu sur les lieux des funérailles, Wangrin menace de faire exhumer le corps à des fins d'autopsie, tout en sachant qu'une telle procédure est sacrilège pour ses hôtes. Il use d'intimidation, de mystification et de chantage pour arracher une autre fortune au fils de feu Brildji. Après avoir mis à couteaux tirés le fils et le frère du défunt, il suggère une solution au problème créé par ses propres soins, en proposant de laisser les biens de Brildji à son fils et de désigner son frère comme nouveau chef. Il revient soumettre son rapport sur le pseudo conflit au commandant, lequel le félicite pour son efficacité et le recommande pour une médaille d'honneur du travail. Dans un tel contexte de duperie et d'abus de pouvoir, certaines autorités traditionnelles en viennent à afficher ouvertement leur hostilité à l'interprète. Lorsque Moukouri est nommé au poste d'adjoint au chef de subdivision et de président suppléant du tribunal de premier degré, les chefs traditionnels de la région se concertent et décident d'entreprendre une démarche auprès de l'administration afin d'obtenir l'annulation de cette nomination, au motif officiel qu'ils ne sauraient être placés sous les ordres d'un de leurs sujets (Moukouri 1963: 113).

\section{L'interprète et les Européens}

La communauté européenne est relativement petite, se composant essentiellement du personnel administratif, d'une poignée de commerçants, de quelques membres des professions libérales, ainsi que de missionnaires. En dehors du personnel administratif, l'interprète entretient peu de rapports avec les Européens en général, entre autres parce que ceux-ci n'ont pas besoin de lui. Contrairement aux administrateurs, dont le séjour est bref et qui ignorent parfois tout de la localité où ils servent, les autres colons sont davantage sédentaires et, en plus de posséder une bonne connaissance des réalités locales, certains d'entre eux apprennent les langues autochtones.

Dans l'ensemble, les rapports entre l'agent colonial européen et l'interprète sont à l'image de ceux qui existent entre tout colon et tout colonisé: le premier se sent investi d'une mission civilisatrice auprès du second, et il adopte à son égard un complexe de supériorité caractérisé entre autres par l'infantilisation, le mépris et le paternalisme. Les membres de la société coloniale sont divisés en deux catégories: les citoyens français et les sujets français (c'est-à-dire la quasi-totalité des autochtones). Les citoyens tutoient les sujets tout en se faisant vouvoyer par ces derniers. Le titre de Monsieur est exclusivement réservé au citoyen. Le sujet est assujetti à l'indigénat, code en vertu duquel tout citoyen est par exemple habilité à 
arrêter, à juger et à punir tout sujet. L'autorité coloniale a particulièrement peu d'estime pour la masse autochtone qui constitue le groupe le moins exposé à la civilisation:

le degré de moralité d'un individu se mesurait d'une part à l'importance des services qu'il avait rendus à la pénétration française et, d'autre part, à la situation géographique de son pays d'origine. C'est ainsi que les plus moraux des hommes étaient les Européens blancs. Après eux venaient progressivement les Martiniquais et Guadeloupéens, puis les Sénégalais autochtones des quatre communes - Saint-Louis, Gorée, Rufisque et Dakar -, les anciens militaires indigènes et enfin, en dernier lieu, le restant de la population. (Hampaté 1992: 49)

La fonction publique reflète la ségrégation qui caractérise la société coloniale. Elle comporte deux cadres différents, dont l'un pour les Européens et l'autre pour les Africains. Aucune familiarité n'est tolérée entre les fonctionnaires des deux cadres. Tous les Africains appartiennent à des corps subalternes, de sorte que "quel que soit son grade, l'agent indigène reste subordonné au fonctionnaire ou agent européen” (Moukouri 1963: 26). Le salaire du plus petit fonctionnaire Européen est en moyenne trente fois supérieur à celui du fonctionnaire autochtone le mieux rémunéré. Au plan professionnel, certains Européens éprouvent de la difficulté à admettre qu'un autochtone, par définition inférieur et à l'esprit primitif, puisse être utile, voire indispensable dans l'appareil administratif où il est censé représenter "moins que la cinquième roue du char" (18). C'est pourquoi, tout en s'appuyant officieusement sur l'interprète pour remplir ses fonctions, l'agent colonial Européen s'attache en public à lui renier la moindre importance: il l'humilie, le brutalise et l'accable de grossières injures (34-35). L'interprète n'a pas d'autre choix que d'endurer stoïquement ce traitement, car toute protestation entraînerait des sanctions, comme l'amputation de salaire pour insubordination ou "mauvaise manière de servir" (ibid.). Tous les administrateurs sont conscients de ce que, en plus de jouer un rôle d'intermédiaire entre eux et les populations, l'interprète est très influent auprès non seulement de ces dernières, mais aussi des chefs, des notables et de l'élite. Certains administrateurs essaient de tirer parti de cette situation en faisant de l'interprète leur meilleur allié et conseiller. En revanche, d'autres en prennent ombrage et confinent l'interprète à des tâches secondaires, lorsqu'ils ne le font pas muter ailleurs. Dans tous les cas, les commandants se méfient de l'interprète et n'hésitent pas de voir sa main derrière toute difficulté qu'ils rencontrent auprès des populations. Ils mettent en place des réseaux parallèles d'informateurs pour tenter de contrer l'influence de l'interprète.

Paradoxalement, devant l'interprète qui, en tant qu'indigène évolué, symbolise pourtant le succès de la mission civilisatrice, l'administrateur colonial semble éprouver un sentiment de malaise et d'insécurité. En effet, il est clair que l'interprète, grâce à ses fonctions, partage l'autorité non seulement du chef traditionnel, mais aussi de l'administrateur européen, ce 
qui fait de lui l'un des plus puissants acteurs de la scène coloniale locale. Du coup, le commandant estime que l'interprète "évolue trop vite" et qu'il constitue "l'élément le plus dangereux de la politique indigène" (Moukouri 1963: 63). On redoute l'impact de son activisme vrai ou imaginé auprès de la population. Il y a risque qu'il devienne le "levain qui [fait] monter toute la pâte" (63). Il est nécessaire de le surveiller et de lui interdire toute initiative politique.

\section{L'interprète et la quête identitaire}

En tant que produit de la colonisation, l'interprète est tiraillé entre la conservation des valeurs traditionnelles et l'occidentalisation. Il essaie de se ménager une place dans les deux mondes. Lors de son initiation, Wangrin se place sous la protection du dieu mythologique Gongoloma-Sooké, qui symbolise le "confluent des contraires", un peu comme la propre personnalité future de Wangrin lui-même. Partout où il est affecté, Wangrin observe les coutumes locales, offrant des sacrifices aux dieux et adhérant à des groupes culturels traditionnels. Il associe à toutes ses initiatives des marabouts et des géomanciens. Plus tard, il imputera sa déchéance à la violation d'une série d'interdits liés aux croyances traditionnelles (Hampaté 1992: 323-327): de passage dans son village, il omet d'aller, avant toute chose, offrir des sacrifices aux mânes de ses ancêtres; il s'aperçoit à la même occasion qu'il a oublié de voyager avec la pierre sacrée qui l'unit à l'esprit de son dieu protecteur; sa voiture écrase un dieu piton; Mme Terreau, sa maîtresse, fait jeter à la poubelle sa pierre sacrée; il sait que son sort est scellé lorsqu'il aperçoit la "tourterelle au cou cerclé de noir" (334), signe annonciateur de sa fin. En même temps qu'il s'attache à des aspects de la société traditionnelle, Wangrin adopte des valeurs occidentales et exploite à son avantage les faiblesses du système colonial. De son côté, Moukouri recherche et obtient la bénédiction du Ngondo (association socioculturelle regroupant les notables de son ethnie) avant de prendre ses fonctions d'administrateur. Dans la vie privée, il se soumet volontiers à l'autorité du chef traditionnel, lequel reçoit pourtant ses ordres officiels de lui.

Évoquant le comportement des collaborateurs autochtones de l'administration coloniale, Moumouni (1964: 60-61) écrit qu'ils sont des "zélés soumis ... inconscients de leur position et de leur rôle dans la société, vides de tout contenu humain, sans vie spirituelle, détachés du peuple, imitateurs du 'Blanc', propagateurs des mythes de la supériorité du 'Blanc' et de l'infériorité congénitale du noir...". Un tel jugement peut s'appliquer de manière générale à Moukouri, et dans une moindre mesure à Wangrin. Si chacun de ces interprètes est plus que conscient de son statut et de son rôle social, et mène une vie spirituelle plus ou moins intense (Wangrin dans un mélange d'islamisme et d'animisme, Moukouri dans le christianisme), l'un et l'autre cherchent à se faire "une place au soleil tout en acceptant la domination", pour reprendre la formule d'Okolo Okonda (1986: 52). Chacun 
d'eux aspire, à sa manière, à exercer un pouvoir aussi grand que celui du commandant. En attendant - ou à défaut - d'y parvenir, ils vont singer le Blanc qui est l'incarnation du pouvoir, tant il vrai, comme le relève Mbembe (2001: 28), que "[c]ommandement itself was simultaneously a tone, an accoutrement, and an attitude". Ainsi, pendant sa première rencontre avec les fonctionnaires indigènes de Dioussola, Wangrin

se comporta comme il l'avait vu faire aux grands chefs blancs-blancs lorsqu'on leur présentait des fonctionnaires: il serra rapidement les mains en souriant plus ou moins largement selon le grade et le cadre auquel appartenait l'intéressé. Il adressa quelques questions rapides et fit de petits éclats de rire... Wangrin se tirait de son rôle à merveille. Excellent mime, il savait imiter à la perfection et pouvait reproduire à volonté tous les gestes, sons et cris qu'il voulait. (Hampaté 1992: 226-227)

Moukouri quant à lui commande de France un livre sur le savoir vivre qu'il étudie religieusement afin de pouvoir acquérir l'art de se comporter à l'occidentale. Lors des soirées mondaines où se retrouvent les Européens, il rase les murs pour épier les invités, dans l'espoir d'observer de près des exemples concrets de bonnes manières. Dans l'exercice de ses fonctions, il choisit de s'exprimer exclusivement en français, y compris dans les situations où il partage la même langue maternelle que ses interlocuteurs (115-116). Il évoque - pour s'en féliciter - un incident au cours duquel sa tante, à la suite d'un litige avec un voisin, se rend à son bureau pour se plaindre; il fait venir un interprète pour l'audience qui se déroule alors entièrement en français, au grand étonnement de sa tante; le soir à la maison, cette dernière lui demandera s'il ne l'avait pas reconnue dans la journée (117). Il explique son attitude par le besoin de transcrire le procès-verbal de l'audience, mais aussi par la nécessité de préserver le prestige de l'administration qui doit strictement se dérouler en français. Pendant une tournée, il étouffe de gêne lorsque, après l'exécution de la Marseillaise, la fanfare entonne un air traditionnel de sa région natale. Il estime que dans ses fonctions officielles il représente la France et non un groupe ethnique africain, fût-ce le sien. Il est soulagé d'apprendre, par la suite, que l'exécution de l'air en question relevait d'une pure coïncidence et n'avait rien à voir avec sa présence. Vers la fin des années quarante, quand les autorités françaises annoncent leur intention d'accorder plus d'autonomie aux colonies, Moukouri "pleur[e] de joie, d'admiration pour la France. Cette France dont les fils métropolitains tels des frères aînés savaient exprimer les sentiments profonds de leurs jeunes frères africains mieux que ces derniers eux-mêmes" (86).

\section{L'interprète et l'idéologie de la colonisation}

L'on ne saurait apprécier le comportement respectif des évolués et des colons sans avoir une certaine idée des fondements et de la nature du projet colonial. La colonisation repose sur une idéologie eurocentrique résultant 
lui-même d'un ethnocentrisme épistémologique (Mudimbe 1988) qui revendique pour l'Occident la paternité exclusive de la science, l'apanage de la raison et le monopole de la 'civilisation'. Inspiré par cette idéologie, le colon perçoit le Noir comme "a being immersed in nature, bad in essence, lazy, impulsive, superstitious, submitted to passion, incapable of reasoning" (Mudimbe 1994: 128), ce qui justifie la mission civilisatrice. L'indigène étant assimilé à un animal, la seule relation que le colon puisse entretenir avec lui en sera une de violence et domination (Mbembe 2001: 27), menant à sa domestication (Mudimbe 1994: 129). En d'autres termes, la mission civilisatrice ne vise pas à faire de l'indigène un être "civilisé" et égal à l'Européen, pour la simple raison qu'il n'existe pas entre eux de "communauté d'essence" (Mbembe 2001: 27). Il s'agit plutôt de dresser l'indigène afin de mieux le contrôler et l'exploiter. Selon Mbembe (ibid.), même les manifestations de sympathie et d'amour participaient de la domination: “the master's/mistress's affection for the animal presented itself as an inner force that should govern the animal. In the Bergsonian tradition of colonialism, familiarity and domestication thus became the dominant tropes of servitude". La société coloniale, née de la violence (celle de l'eurocentrisme et de la conquête), se caractérise par le règne de l'arbitraire, de l'impunité et de la brutalité. Elle apparaît comme une véritable jungle où le plus fort impose sa loi. À ce sujet, Memmi (1985: 41) parle d'une "pyramide des tyranneaux" où "chacun, socialement opprimé par un plus puissant que lui, trouve toujours un moins puissant pour se reposer sur lui, et se faire tyran à son tour". Du haut de la pyramide, le colon exerce le commandement, c'est-à-dire "the right to demand, to force, to ban, to compel, to authorize, to punish, to reward, to be obeyed - in short, to enjoin and direct" (Mbembe 2001: 32). La fin justifiant toujours les moyens, il viole au besoin ses propres lois, tout comme il n'hésite pas à prétexter de la raison d'État pour exercer le droit à des fins privées. En dessous des colons se trouvent les indigènes évolués, qui cherchent à tirer le meilleur parti des miettes de pouvoir dont ils disposent. Ils "forment une catégorie de colonisés qui prétend échapper à sa condition politique et sociale. Mais choisissant de se mettre pour cela au service du colonisateur et de défendre exclusivement ses intérêts, ils finissent par en adopter l'idéologie, même à l'égard des leurs et d'eux-mêmes" (Memmi 1985: 41). Leurs efforts pour accéder au sommet de la pyramide sont sans cesse annihilés par les colons, lesquels y voient une grave menace à leur statut de privilégiés. De guerre lasse, l'évolué fait le deuil de son désir d'assimilation et se tourne vers des valeurs-refuges (Memmi 1985: 117), à savoir la famille, le clan, les pratiques et les croyances traditionnelles. Les privilèges et les honneurs dont il jouit parmi les siens suscitent en lui un sentiment de contentement, voire une illusion de réussite, qui masquent à la fois l'échec de sa tentative d'accession à la classe supérieure et son incapacité de revendiquer plus de droits et de justice pour lui et les autres autochtones.

À la lumière de ce qui précède, il apparaît que le mépris du colonisé et son dénigrement systématique relèvent d'une stratégie de survie chez le 
colon: reconnaître la moindre qualité à l'indigène reviendrait à remettre en question sa propre légitimité, et le considérer comme un être humain obligerait à lui concéder des droits, ce qui nuirait à sa domestication et à son exploitation. Le commandant a donc tout intérêt à humilier publiquement l'interprète et à étouffer chez lui toute velléité d'émancipation. En ce qui concerne le colonisé évolué, dont l'interprète constitue le modèle type, le maître mot semble être la satisfaction des intérêts personnels. Pour ce faire, il contribue volontiers à la propagation de l'idéologie eurocentrique des colons, ainsi qu'au maintien des iniquités du régime colonial. Le colon est pour lui un "modèle prestigieux" auquel il cherche à ressembler, le mariage mixte étant "le terme extrême de cet élan chez les plus audacieux" (Memmi 1985: 137). Ce reniement de soi conduit en fin de compte à sa perte, ainsi que l'apprendra Wangrin au terme de son concubinage avec Mme Terreau. Par ailleurs, l'attachement de l'évolué aux valeurs traditionnelles s'avère superficielle et opportuniste, comme l'illustrent l'adhésion de Wangrin au waaldé et la pseudo-soumission de Moukouri au Ngondo et aux chefs traditionnels. L'évolué se range résolument du côté des forces réactionnaires de la société coloniale: souscrivant à l'idéologie paternaliste du colon, Moukouri s'emploie à contrer les nationalistes qui dénoncent le régime colonial et en réclament la fin; il est pour une approche graduelle de l'autodétermination, estimant que les autochtones ne sont pas encore 'prêts' à prendre en main la destinée de leur pays; il prône une stricte séparation des revendications syndicales et politiques, une démarche qui aboutirait en fait à la division et à l'affaiblissement de l'élite locale; il est également contre l'excès de zèle en matière de lutte syndicale; il tente de s'opposer à de nombreux changements sociopolitiques qu'il juge trop rapides ou révolutionnaires; cette attitude lui vaudra d'être "traité de lâche et de vendu" (Moukouri 1963: 90-91). Bref, l'interprète et les autres évolués forment la classe que Frantz Fanon (1961) désignera sous le nom de bourgeoisie nationale, et dont la caractéristique dominante est qu'elle est le principal suppôt du néocolonialisme.

\section{Conclusion}

Tel qu'il se dégage des deux ouvrages examinés ci-dessus, le portrait de l'interprète est celui d'un personnage aux traits plutôt contradictoires, ce qui n'est autre que le reflet de la société dont il est le produit. Témoin privilégié de la violence perpétrée par le colon à l'endroit de la population, et conscient des conséquences de la moindre résistance à l'autorité coloniale, il se range par réalisme du côté du plus fort et s'efforce de s'identifier à ce dernier. En même temps, il s'aménage une place de choix parmi les autochtones, en veillant toutefois à ne pas sacrifier ses intérêts personnels. Bien qu'il soit craint et admiré par les autochtones, et fort utile au commandant, il n'est véritablement accepté par aucun des principaux groupes en présence dans la société coloniale. En s'imposant comme l'interlocuteur incontournable des 
autorités traditionnelles et administratives, il apparaît aux yeux des unes et des autres comme un usurpateur.

Dans ces conditions, sa tâche ne peut être qu'ingrate: comme le souligne Moukouri, lorsque la médiation de l'interprète mène au dénouement heureux d'une crise, sa contribution n'est jamais reconnue; la haute administration salue la grande compétence du commandant, tandis que la population félicite le chef et les notables pour leur "efficace intervention ou représentation" (1963: 20-21); par contre, en cas d'échec, l'interprète est le bouc émissaire tout désigné, chacune des parties l'accusant de l'avoir mal servie; de même, lorsque des mesures impopulaires sont adoptées, les critiques à l'endroit de l'administration n'épargnent guère l'interprète, car il est alors associé à son commandant. Pour compliquer la situation, les avantages liés à la fonction d'interprète attirent dans le métier de nombreux fonctionnaires véreux qui s'intéressent avant tout à la recherche de rente. Cette ruée vers la profession d'interprète est facilitée dans certaines régions par l'absence de critères stricts de sélection, le résultat en étant entre autre la réputation d'incompétence qui finit par déteindre sur l'ensemble du corps. Le climat de méfiance qui caractérise les rapports entre l'interprète et les autorités (administratives et traditionnelles), la mauvaise réputation découlant de l'affairisme de certains interprètes, ainsi que la vulgarisation de la scolarisation (qui favorise le contact direct entre administrateur et administré), vont ultimement se conjuguer pour sonner le glas de la profession: quelques années après la Deuxième Guerre mondiale, de nombreux territoires coloniaux éliminent de leur fonction publique le cadre des interprètes, et la plupart de ces derniers se voient confier d'autres tâches administratives ou choisissent de démissionner pour embrasser de nouvelles carrières.

\section{Bibliographie}

Fanon, Frantz (1961). Les damnés de la terre. Paris: Maspéro.

Hampaté Bâ, Ahmadou (1992). L'étrange destin de Wangrin ou les roueries d'un interprète africain. Paris: Éditions 10/18.

Manga Mado, Henri-Richard (1970). Complaintes d'un forçat. Yaoundé: Éditions CLE.

Mbembe, Achille (2001). On the Postcolony. Berkeley: University of California Press.

Memmi, Albert (1985). Portrait du colonisé. Portrait du colonisateur. Paris: Gallimard.

Mopoho, Raymond (2001). "Statut officiel de l'interprète dans l'administration coloniale en Afrique française". Meta 46(3), 615-626.

Moukouri, Jacques Kuoh (1963). Doigts noirs. Je fus écrivain interprète au Cameroun. Montréal: Éditions à la page.

Moumouni, Abdou (1964). L'éducation en Afrique. Paris: Maspéro.

Mudimbe, Valentin Yves (1988). The Invention of Africa: Gnosis, Philosophy, and the Order of Knowledge. Bloomington: Indiana UP.

Mudimbe, Valentin Yves (1994). The Idea of Africa. Bloomington: Indiana UP. 
Okolo Okanda, W'oleko (1986). Pour une philosophie de la culture et du développement. Kinshasa: Presses universitaires du Zaïre.

Raffenel, Anne (1846). Voyage dans l'Afrique occidentale, exécuté en 1843 et 1844 par une commission composée de MM. Huard-Bessinières, Jamin, Raffenel, Peyre-Ferry et Pottin-Patterson. Paris: Arthus Bertrand. 